\title{
VISUALIZATION OF UNDEREXPANDED JET FLOWS OVER A PLATE BY PRESSURE - SENSITIVE PAINT TECHNIQUE
}

\author{
M. K. IBRAHIM*
}

\begin{abstract}
A pressure measurement technique which employs the tools of molecular spectroscopy has recently received considerable attention in the aerodynamic community. Measurements are made via oxygen sensitive molecules attached to the surface of interest as a coating, or paint. The pressure sensitive paint (PSP) measurement technique is now commonly used in stationary wind tunnel tests for both steady and unsteady tests. In the present research, low-cost PSP system is developed and experiments are performed on sonic under-expanded jet issuing from a converging nozzle and impinging on a flat plate at different oblique incidences. Pressure measurements on the plate surface are acquired by pressure transducers at discrete points and by PSP intensity based measurement method. The paint employed in the present research is Bathophen Ruthenium Chloride ( $\mathrm{Ru}\left(\mathrm{ph}_{2}\right.$-phen) or $\mathrm{Ru}(\mathrm{dpp})$ ) dissolved in dichloromethane while Silicon is used as a binder. Pressure transducer readings are used to calibrate the image obtained by PSP, a process known as "in- situ" calibration. Commercially available, inexpensive Charge-Couple Device (CCD) camera is used to capture the PSP images. Corrections due to darkness are applied via locally-developed post processing software. The images obtained by the developed low-cost PSP system show high spatial resolution of the pressure distribution compared with normal measurement techniques. The results can be used as validation test cases for computational fluid dynamics (CFD) solvers.
\end{abstract}

\section{KEY-WORDS}

Pressure Sensitive Paints (PSP), Under-expanded Sonic Jet, Jet-Plate Impingement, Bathophen Ruthenium Chloride, and in- situ Calibration Technique.

\section{NOMENCLTURE}

$\gamma \quad:$ Specific heat ratio

$\lambda \quad$ : Luminescent emission wave length

* Lecturer, Aerospace Engineering Department, Faculty of Engineering, Cairo University, Giza, 12613 Egypt. email: mkhalil@eng.cu.edu.eg. Currently Research Associate, Aerospace Engineering Department, Graduate School of Engineering, Nagoya University, Furo-cho, Chikusa-ku, Nagoya 464-8603, Japan. email: khalil@nuae.nagoya-u.ac.jp 
CCD : Charge-Coupled Device

CFD : Computational Fluid Dynamics

ICCD : Intensified Charge-Coupled Device

NPR : Nozzle Pressure Ratio $=P_{t} / p_{\infty}$

PMT : Photomultiplier Tubes

PSP : Pressure Sensitive Paints

TSP : Temperature Senstive Paints

$\theta \quad$ : Plate inclination angle in degrees

$A \quad$ : Stern-Volmer relation first constant

$B \quad$ : Stern-Volmer relation second constant

I : Luminescent intensity

$I_{r e f}$ : Luminescent intensity at a reference condition

$M_{j} \quad$ : Fully expanded Mach number

$p \quad$ : pressure

$p_{\infty} \quad$ : Ambient pressure

$P_{t} \quad$ : Chamber total pressure

$p_{r e f}:$ Air pressure at a reference condition

$R u\left(p h_{2}-p h e n\right)$ :Bathophen Ruthenium Chloride

\section{INTRODUCTION}

Quantitative measurements of surface pressure in wind tunnels are essential in the understanding of aerodynamic performance of flight vehicles. Pressure data is required to determine the distribution of aerodynamic loads for the design of a flight vehicle. Pressure measurements provide critical information on important flow phenomena such as shocks, flow separation and boundary-layer transition.

Traditionally [1], surface pressure is measured by utilizing a pressure tap or an orifice at a location of interest connected through a small tube to a pressure transducer. Hundreds of pressure taps are needed to obtain an acceptable pressure field on a complex aircraft model. Manufacturing, tubing and preparing such a model for wind tunnel testing is costly. For thin models such as supersonic transports, military aircraft and small fan blades, installation of a large number of pressure taps ultimately limits the spatial resolution of measurements such that some details of a complex field cannot be revealed.

Since the 1980s, new technologies using optical sensors for measuring surface pressure have been developed based on the quenching mechanisms of luminescence. 
These luminescent molecule sensors are called pressure sensitive paints(PSP). Compared with conventional techniques, PSP technology offer a unique capability for noncontact, full field measurements of surface pressure on complex aerodynamic model with much higher spatial resolution and lower cost. Therefore this technology provide a powerful tool for experimental aerodynamicists to gain a deeper understanding of physical phenomena occurring in complex flows around flight vehicles [1].

PSP use luminescent molecules as probes that are incorporated into a suitable polymer coating on the surface of an aerodynamic model. Generally, the luminophore and polymer binder in PSP can be dissolved in a solvent. The resulting paint can be applied to the surface of the model using any painting technique. After the solvent evaporates, solid polymer coating in which the luminescent molecules are immobilized remains on the surface, when light of proper wavelength illuminates the paint, the luminescent molecules are excited and the luminescent light of a longer wavelength is emitted from the excited molecules [1]. Fig. 1 shows a schematic of conventional polymer PSP. The luminescent emissions from a paint layer can be affected by certain physical processes. The main photo-physical process in PSP is oxygen quenching that causes a decrease in luminescent intensity as the partial pressure of oxygen or air pressure increases. Therefore the polymer binder must be oxygen permeable. In fact PSP is an oxygen-sensitive sensor.

A measurement system for PSP is generally composed of paint, illumination light, photo-detector, and data acquisition/processing unit. Photodetectors are usually scientific - grade charge-coupled device (CCD), intensified charge-coupled device (ICCD), or photomultiplier tubes (PMT). Usually these photo-detectors are very expensive and considered the critical issue in PSP technology. Thanks for the advances in digital image technology which developed commercial digital cameras with high resolution and low cost. The main objective of the present study is to use a low-cost and commercial digital camera in the PSP technique and apply this to under-expanded sonic jet-plate interaction problem.

There are many applications where the under-expanded sonic jet impingement on a solid boundary or object is encountered. In aeronautics and space sciences there are wide varieties of such applications like multistage rocket separation. Other applications of jet-plate impingement include but are not limited to deep-space docking, spacemodule attitude-control thruster operation, lunar and planetary landing and take-off, jet engine exhausts impingement of STOVL (short take-off and vertical landing aircraft), gas-turbine blade failure, gun-muzzle blast impingement, shock-impingement heating and terrestrial rocket launch, as well as many other engineering applications in lasers, material processing and manufacturing [2].

These impingement flows are generally found to be extremely complex. They contain mixed subsonic and supersonic regions, elaborated interacting shock and expansion systems, highly non-uniform upstream flows, shock-boundary layer interaction, turbulent shear and instance of major instabilities, sound-producing oscillations which is generally explained using feedback loop mechanisms. Extensive efforts are needed to 
understand the undergoing phenomena and control its undesirable produced sound. This can in turn be beneficial in the control of other annoying noise sources in daily life.

Lamont and Hunt [2] did an extensive flow-field study of single jet impingement of a flat plate by means of mean pressure measurement on the flat plat surface and shadowgraph visualizations. Fuji et al. [3] tried to use CFD (Computational Fluid Dynamics) and PSPs (pressure sensitive paints) to understand the interaction mechanisms neverthelees, although their CFD results do agree qualitatively well with the mean flow experimental data, the quantitative comparison is still under way. Crafton et al. [4] studied the same impingement problem with both PSP and temperature sensitive paint (TSP). The present study focuses on the impingement of jet-plate pressure distribution visualization by developing low-cost PSP technique.

\section{BASIC PHOTOPHYSICS OF PRESSURE SENSITIVE PAINTS}

As mentioned above, pressure sensitive paint (PSP) is based on oxygen quenching process of luminescence which is a reversible process in molecular photoluminescence. Photoluminescence is a mechanism, in which the molecule can lose the excess energy by emitting photons, and return to the ground electronic state. In this process, lower energy photons are emitted; i.e., the emitted luminescence is red-shifted compared with the excitation luminescence. Fluorescence is the luminescence emission in the order of $10^{-8} \mathrm{sec}$. due to a singlet-singlet transition, while phosphorescence is a delayed emission in the order of $10^{-2}-100 \mathrm{sec}$. due to a triplet-singlet transition. The different energy levels and photo-physical processes of luminescence for a simple luminophore can be clearly described by Jablonski energy-level diagram as shown in Fig. 2 [1]. An alternative transition to the ground state is made by a collision with the oxygen molecule. In this case instead of emitting a photon, the excess energy of the probe molecule is absorbed by oxygen during collision deactivation. The process is called dynamic quenching. As the number of oxygen molecules increase in a given volume, the frequency of collision deactivation also increase, which in turn lower the luminescence

The luminescence of a molecule, when exposed to oxygen, can be modeled in many cases by the Stern-Volmer relation [5].

$$
\frac{I_{r e f}}{I}=A+B \frac{p}{p_{r e f}}
$$

where $I_{r e f}$ and $p_{\text {ref }}$ are luminescent intensity and air pressure at a reference condition, respectively. The Stern-Volmer coefficient $A$ and $B$, which are temperature-dependent due to the thermal quenching, are experimentally determined by calibration. Thermal quenching is the mechanism in temperature sensitive paints (TSP) that reduces the luminescent intensity as temperature increases. Theoretically speaking, the intensity $I_{r e f} / I$ can eliminate the effects of non-uniform illumination, uneven coating and non homogenous luminophore concentration in PSP. In typical tests in a wind tunnel, $I_{\text {ref }}$ 
is taken when the tunnel is turned off and hence it is often called wind-off image or intensity; likewise, I is called the wind-on image or intensity.

Reference [1] is considered and excellent review of both PSP and TSP measurement techniques and calibration methods. In the present research the intensity-based method is employed as the intensity of the luminescence is measured directly by CCD. The Calibration employed in the present study is the in-situ calibration technique. Calibration of PSP in situ is done by fitting or correlating the luminescent intensity to the pressure tap data at a number of suitably distributed locations. In a sense, in-situ PSP calibration eliminates the systematic error associated with the temperature effect and the illumination change by absorbing it into an overall fitting error.

\section{EXPERIMENTAL APPARATUS}

\subsection{Test Facility}

Experiments were conducted in the open jet facility at the Department of Aerospace Engineering, Faculty of Engineering, University of Cairo, as shown in Fig. 3 and Fig. 4. The nozzle employed in the present study to produce the primary jet flow is a sonic convergent nozzle of throat diameter $10 \mathrm{~mm}$. The nozzle is attached to a cylindrical plenum chamber that has a diameter of $153 \mathrm{~mm}$ and a length of $400 \mathrm{~mm}$. High-pressure air is supplied from a tank with a volume of $2 \mathrm{~m}^{3}$ stored at a pressure of $10 \mathrm{kgf} / \mathrm{cm}^{2}$ that is connected to the plenum chamber via a 1 inch inner diameter high-pressure pipe. A 10 $\mathrm{mm}$ thick Aluminum flat plate with width of $100 \mathrm{~mm}$ and length of $200 \mathrm{~mm}$ is employed as the impinging plat in the present study. The coordinate system employed here considers the nozzle center as the origin, while the $y$ - and $x$-axes are along the jet axis and the traverse direction of the plate, respectively. Twenty pressure taps were used to measure the pressure on the plate centerline, where the jet impinges the plate, using a single pressure transducer for in-situ PSP calibration technique.

\subsection{Baseline Jet}

The fully-expanded Mach number $M_{j}$ is uniquely related to the pressure ratio, $P_{t} / p_{\infty}$, which is also called the nozzle pressure ratio or $N P R$, through the following equation:

$$
M_{j}=\left\{\left[\left(\frac{P_{t}}{P_{\infty}}\right)^{\frac{\gamma-1}{\gamma}}-1\right] \frac{2}{\gamma-1}\right\}^{0.5}
$$

where $P_{t}$ is the chamber pressure and $p_{\infty}$ is the ambient pressure. The baseline jet employed in this study is the same jet used by Obase and Nakamura [6] which has $P_{t} / p_{\infty}=5$, that corresponds to $M_{j}=1.71$; this is a typical under-expanded sonic jet. The Mach number in the plume of an under-expanded sonic jet can become actually higher than $M_{j}$. The pressure as well as the Mach number overshoot or undershoot thier corresponding fully-expanded values as the flow passes through the shock cell 
system. Note that $M_{j}$ is nothing more than the exit Mach number of a fictitious nozzle, at whose exit the flow expands up to the given NPR. In other words, it is an average jet Mach number in the region along the jet axis where the flow oscillates. Fig. 5 shows a Schileren photograph of the baseline jet and its first shock cell structure.

\subsection{Pressure Sensitive Paint}

A typical PSP is prepared by dissolving a luminescent dye and a polymer binder in a solvent; the order of mixing the components and the relative concentration of the components may change the characteristics of the paint [1]. Chlorinated organic solvents such as dichloromethane have been used for making PSP. The selection of the polymer binder for PSP is important, which should be based on a balanced consideration of its oxygen permeability, temperature effect, humidity effect, adhesion, mechanical stability, photo-degradation, and other properties. Silicone rubber, GE RTV 118, silica gel and sol-gel-derived coating have been used as binders for PSP.

In the present research the binder doesn't mix with the luminescent dye. After painting the plate model with a primer paint to reduce the reflection of the excitation light, GE RTV 118 silicon is dissolved in dichloromethane and the resulting mixture is used to paint the model with thin layer of this mixture using air brush or painting gun. Finally the luminophore is attached to this sticky silicon mixture. The major features of this method are as follows; 1) easy to apply, 2) the model can be re-painted, 3) ensure that oxygen is directly accessible to the luminophore which increases the sensitivity and response. The issue here is the selection of the silicon which possesses the required mechanical properties to hold the luminophore in place. The above mentioned painting method was proposed by Kurita et al. [7] and applied to a model in a hybersonic wind tunnel where the density is very low.

Table 1 lists some PSP formulations along with their spectroscopic properties and the Stern-Volmer coefficients A and B as in Eq. (1) [1]. In the present study Bathophen Ruthenium Chloride ( $\mathrm{Ru}\left(\mathrm{ph}_{2}\right.$-phen) or $\left.\mathrm{Ru}(\mathrm{dpp})\right)$ is selected as luminophore because it has been used extensively with PSP technology. Fig. 6 shows the chemical structure, absorption and emission spectra of Bathophen Ruthenium Chloride. The temperature sensitivity of the luminophore is $-1.1 \% \mathrm{~K}$ at $293 \mathrm{~K}$ [7]. The effect of temperature was not investigated in the current work. The Bathophen Ruthenium Chloride is dissolved in dichloromethane with ratio $40 \mathrm{ml}$ of dichloromethane to $28 \mathrm{mg}$ of the Bathophen Ruthenium Chloride. The model is painted with the resulting mixture, after being coated with silicon-based mixture as mentioned above, using a painting gun.

\subsection{Pressure Sensitive Paint Hardware and Software}

The photo-detector employed here is a CCD camera, CANON EOS D300 with resolution of $3072 \times 2048$ pixels. UV lamp is used as the source of excitation light. The image capture rate is one frame per $1 / 4 \mathrm{sec}$. Experiments show that the capture rate is highly dependent on the excitation light. The camera is completely controlled through 
PC through serial port using Canon Remote Capture software. In all experiments the luminescence that CCD camera can detect was filtered to prevent unnecessary illuminating luminescence. The filter is a long pass filter, which can pass luminescence with a wave-length longer than $\lambda=580 \mathrm{~nm}$ at $80 \%$ intensity.

The basic processing procedure in the intensity-based method of PSP is by calculating the ratio between the wind-on image and the wind-off reference image to correct the effects of non-homogenous illumination, uneven paint thicknesses and non-uniform luminophore concentrations. However, this proportioning procedure is complicated by model deformation induced by aerodynamic loads, which results in misalignment between the wind-on and wind-off images. This effect was of great effect in the present research when thin plate model was used. By increasing the plate thickness and stiffening the support system one can reduce this deformation, nevertheless it can not be completely removed and produces noise in the resulting image. Therefore additional correction procedures are required to eliminate the error sources associated with model deformation. These correction procedures are not implemented in the present study. The temperature effect of PSP and self-illumination noise are not implemented here as well. Noise caused by darkness is reduced in the current work by subtraction dark image from the data image, besides employing a simple transformation technique to reduce the noise due to flow-induced vibrations of the plate.

Fig. 7 shows a data processing flow chart developed for the present intensity-based method of PSP with CCD camera. A code is developed for post-processing and calibration. Simultaneous pressure measurements and image captureing processing capability is included in this post-processing code for online processing of PSP images.

\section{RESULTS AND DISCUSSIONS}

The raw images of wind-off and wind-on are shown in Fig. 8 for the case of $\theta=0^{\circ}$. The four small, black circles on the corners of the image represent the fixation screws. The area of interest was picked up from these photos and was input to the post-processing code discussed above. One of the outputs of the post-processing code is the intensity ratio image and is shown in Fig. 9. The signature of the shock cell structure of the jet on the plate is clearly shown where the red areas represent jet expansion regions and the blue areas represent compression regions. The free-shear layer of the jet is clearly identified on the plate. This was not clearly shown in the previous work of under-expanded jet-plate impingement. This reflects the high sensitivity of the present method to capture subsonic pressure variation.

The noise, shown in Fig. 9, could be attributed to many factors which include but are not limited to the following: First, flow-induced vibrations of the plate; thus the wind-off and wind-on images can not simply correspond pixel by pixel. Hence image registration technique [1] is needed to reduce the effect of misalignment. Simple transformation procedures are implemented in the present work but are still not sufficient in ensuring good alignment. Second, the optical filter implemented here might be a source of such 
noise because it passes other interfering luminescences which come from reflection or background. Third, the light source could have small percent of red luminescence which is considered as noisy luminescence. Fourth, the present camera has an effective 12-bit resolution. High resolution cameras for example 14- or 16-bit will improve the quality of the image.

The acquired image can be calibrated using in-situ technique. PSP data was collected and averaged around the taps' points (that were used for pressure mesurement). Fig. 10 shows a curve fit of the intensity as a function of pressure. The general trend is shown as a solid line while the circles are the pressure taps data. The scattering of the pressure taps data might be due to insufficient averaging of PSP points. The present PSP method has a high Stern-Volmer B factor of 0.98 , this value is higher than the value listed in Table 1. This means that the present method might be suitable for subsonic flows.

Fig. 11 shows the calibrated PSP image. The plate surface pressure distribution along the jet axis is shown in Fig. 12. There is a good agreement between the PSP results and the pressure taps measurements. Fig. 13 and 14 shows the intensity of PSP images for $\theta=10^{\circ}$ and $\theta=20^{\circ}$, respectively. Again, the flow-induced vibrations are strongly present in these cases and noise is higher, however the jet effect on the plate is shown clearly in both images.

\section{CONCLUSION}

The surface pressure due to under-expanded sonic jet and plate impingement was visualized by low-cost PSP intensity-based method. In-situ calibration technique was employed in the present study to correlate the captured PSP luminescence with the measured pressure using taps. Post-processing code was developed to get an online PSP image and speed up the development and/or analysis process. Noise caused by darkness is reduced by subtracting dark image from data image, while the noise due to flow-induced vibrations of the plate was reduced by employing a simple transformation technique. The results show good quantitative agreement between PSP measurements and pressure taps measurements. The noise in PSP image is attributed to optical filter, misalignment between wind-off and wind-on image due to model vibration and excitation light noise. further correction procedures for camera non-linearity, filter noise, image registration technique and temperature effect are needed to get highquality PSP images which can be integrated to calculate the areodynmaics forces and can be used as well for CFD validations.

\section{ACKNOWLEDGMENT}

The author would like thank Applied Science International', LLC,(ASI) for the partial financial support during the term of this project. The author would also like to thank

\footnotetext{
${ }^{1}$ www.appliedscienceint.com
} 
Dr. Mitsuru Kurita of Japan Aerospace Exploration Agency (JAXA) for his valuable and sincere advice to the present research as well as Eng. Mohamed Samir El-Attal of Valeo Interbranch Automotive Software, Egypt for his sincere and valuable help.

\section{REFERENCES}

[1] T. Liu and J. P. Sullivan, Pressure and Temperature Senstive Paints Springer, 2005.

[2] P. J. Lamont and B. L. Hunt, Underexpanded Axisymmetric Jets Impinging on Flat Plates, Journal of Fluid Mechanics, Vol. 100-2, pp 471-511, 1980

[3] Kozo Fuji, Nobuyuki Tsuboi, and Nobuyoshi Fujimatsu, Visualization of Jet Flows over a Plate by Pressure Sensitive Paints Experiments and Comparison with CFD, Ann. N. Y. Acad. Sci. 972, pp 265-270, 2002.

[4] Crafton J., Lachendro N., Guille M., Sullivan J. P., Jordan J., Application of Temperature and Pressure Senstive Paint to an Obliquely Impinging Jet, AIAA Paper 99-0387, 1999.

[5] Morries, M. J., Danovan, J. F., Kegelman, J. T., Schwab, S. D., Levy, R. L. and Crites, R. C. Aerodynamic Application of Pressure Senstive Paint, AIAA Journal, Vol. 31, No. 3, pp. 419-425, 1993.

[6] K. Obase and Y. Nakamura, Aerodynamic and Aeroacoustic Interactions of a HighSpeed Jet with a Flat Plate, AIAA-2004-2404, 2nd AIAA Flow Control Conference, Portland, Oregon, June 28-1, 2004

[7] Mitsuru Kurita, Takumi Okada, Ryo Sakuma, and Yoshiyaki Nakamura, Pressure and Temperature Senstive Paint Measurement in Shock Tunnel, Journal of the Visualization Society of Japan, Vol. 22, No. 1, 2002. 

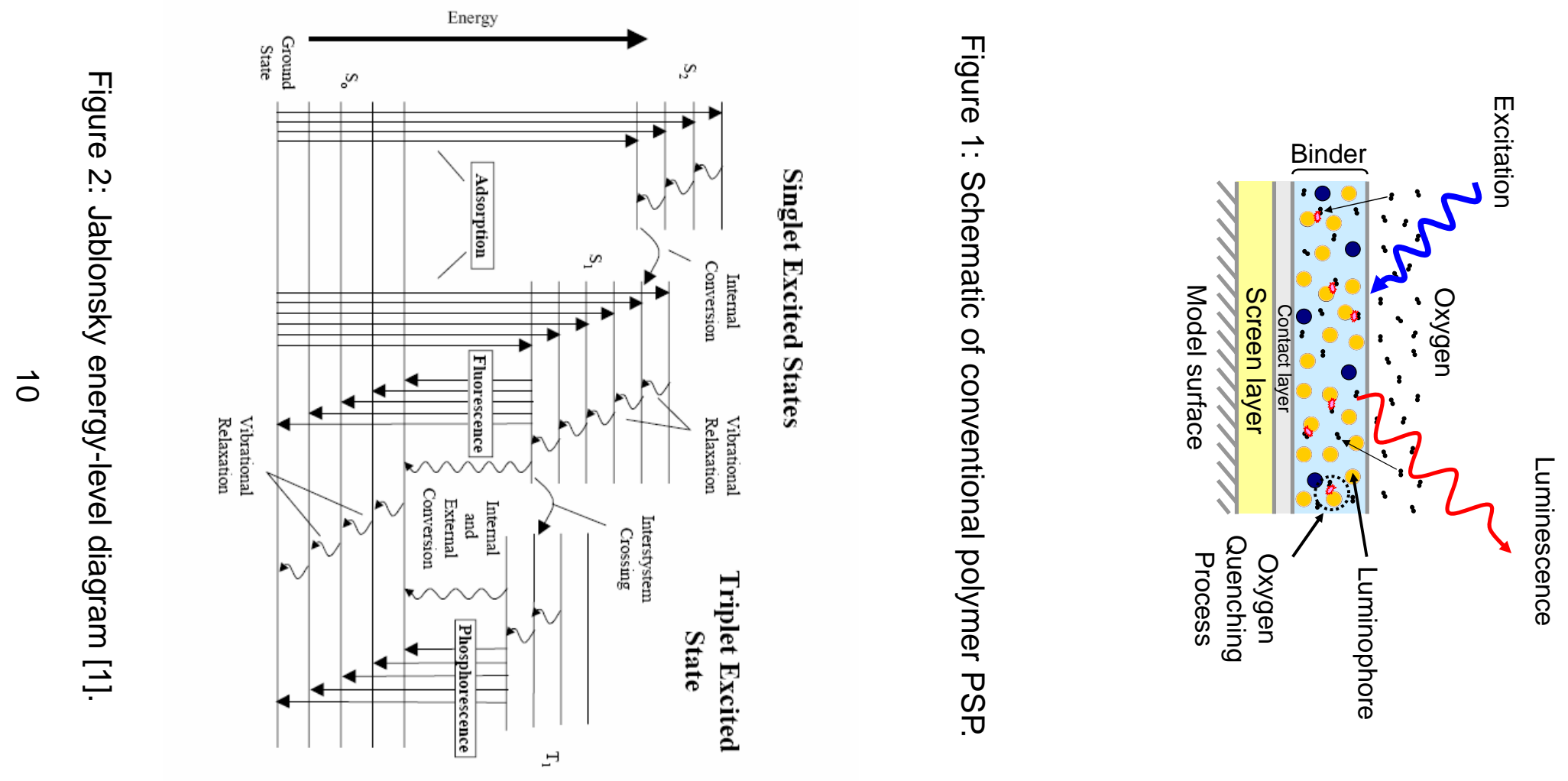

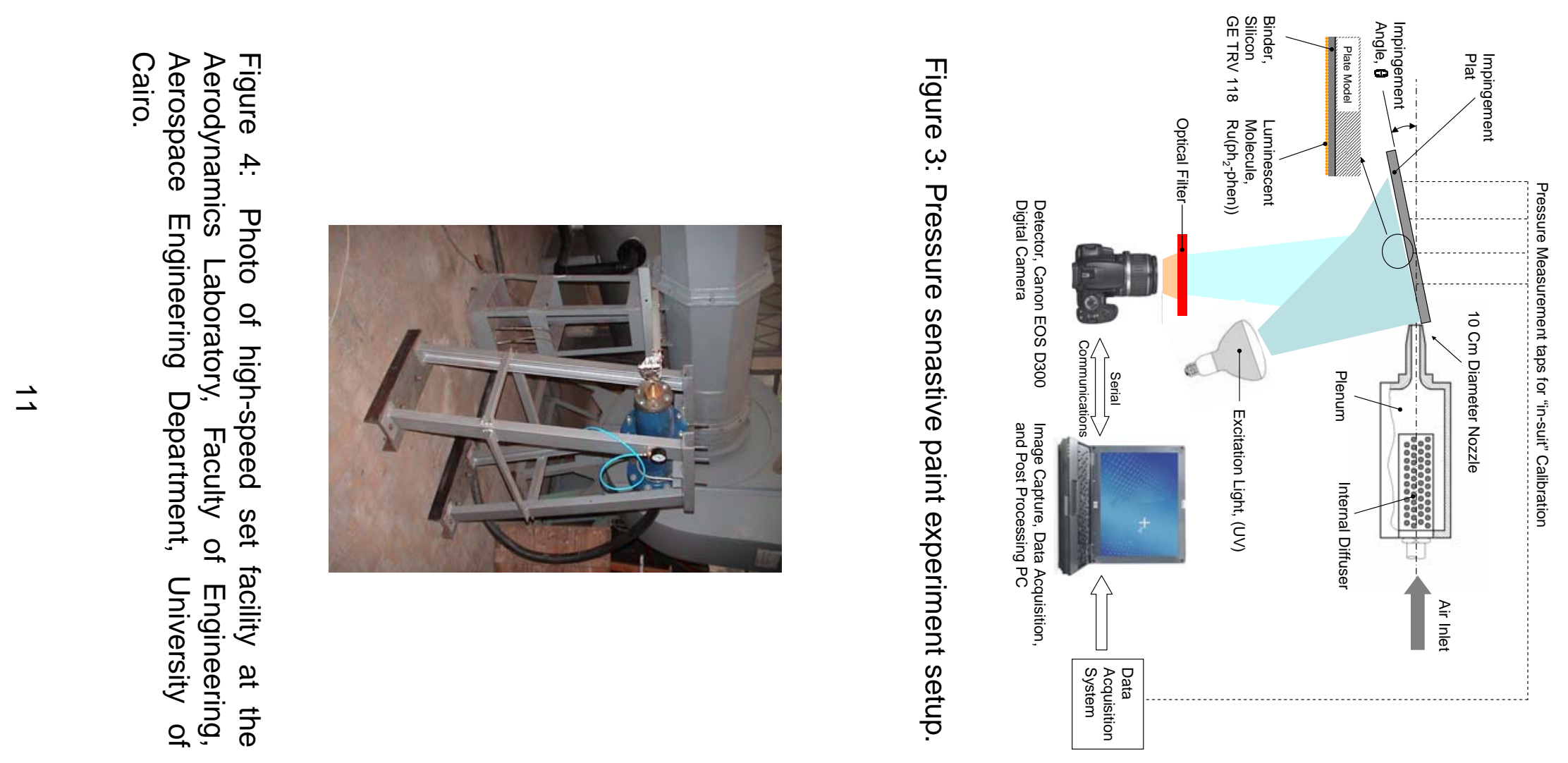

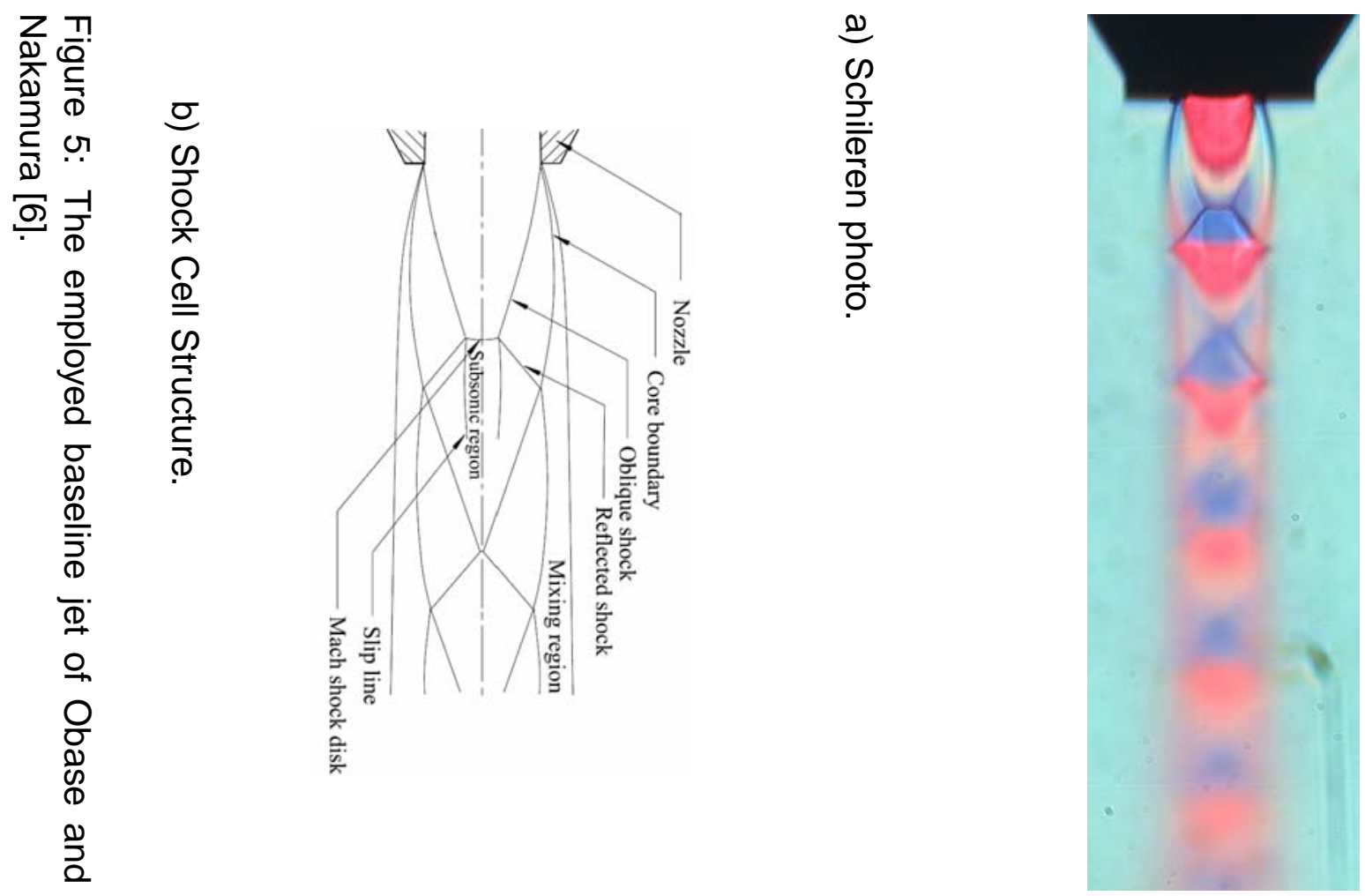
Table 1: Typical Pressure Senstive Paints [1].

\begin{tabular}{|c|c|c|c|c|c|c|c|c|c|}
\hline \multirow[b]{2}{*}{ Luminophore } & \multirow[b]{2}{*}{ Binder } & \multirow[b]{2}{*}{$\begin{array}{l}\text { Excitation } \\
\text { wave- } \\
\text { length } \\
{[\mathrm{nm}]}\end{array}$} & \multirow[b]{2}{*}{$\begin{array}{l}\text { Emission } \\
\text { wave- } \\
\text { length } \\
{[\mathrm{nm}]}\end{array}$} & \multicolumn{2}{|c|}{$\begin{array}{l}\text { Stern-Volmer } \\
\text { coefficients }\end{array}$} & \multirow[b]{2}{*}{$\begin{array}{l}\text { Lifetime } \\
\text { at room } \\
\text { temp. } \\
\text { (micro } \\
\text { sec) }\end{array}$} & \multirow[b]{2}{*}{$\begin{array}{l}\text { Temp. } \\
\text { coeff. } \\
\left(\% /{ }^{\circ} \mathrm{C}\right)\end{array}$} & \multirow[b]{2}{*}{ Reference } & \multirow[b]{2}{*}{$\begin{array}{l}\text { Purchase } \\
\text { source }\end{array}$} \\
\hline & & & & A & B & & & & \\
\hline $\mathrm{H}_{2} \mathrm{TSPP}$ & silica gel & 400 & 650,709 & 0.58 & 0.42 & & & Wan(1993) & Prophyrin \\
\hline $\mathrm{H}_{2}\left(\mathrm{Me}_{2} \mathrm{~N}\right) \mathrm{TFPP}$ & silica gel & 400 & 650 & 0.43 & 0.56 & & $\sim 0$ & Wan(1993) & Prophyrin \\
\hline $\mathrm{H}_{2} \mathrm{TCPP}$ & silica gel & 410 & 709 & 0.40 & 0.61 & & & Wan(1993) & Prophyrin \\
\hline $\mathrm{H}_{2} \mathrm{TNMPP}$ & silica gel & 420 & 661,714 & 0.43 & 0.60 & & $\sim 0$ & Wan(1993) & Aldrich \\
\hline $\mathrm{H}_{2}$ TTMAPP & silica gel & 410 & 653,710 & 0.40 & 0.60 & & & Wan(1993) & Aldrich \\
\hline Perylene dibutyrate & silica gel & 457 & 520 & 0.33 & 0.67 & 0.013 & 4.50 & Burns(1995) & Pylam \\
\hline Perylene dye & silica gel & 480,530 & 550,570 & 0.47 & 0.53 & & 0.35 & Wan(1993) & Aldrich \\
\hline \multirow[t]{3}{*}{ PtTFPP } & silica gel & 390 & 650 & 0.27 & 0.72 & 50.0 & -2.1 & Wan(1993) & Porphyrin \\
\hline & $\begin{array}{l}\text { DuPont } \\
\text { Chrom. }\end{array}$ & & & 0.50 & 0.52 & & -1.80 & Burns1995) & \\
\hline & Polystyrene & & & 0.29 & 0.69 & & -4.30 & & \\
\hline PtTFPP & FEM & 390 & 650 & 0.17 & 0.83 & & -1.40 & & $\begin{array}{l}\text { NASA } \\
\text { Langley }\end{array}$ \\
\hline PtTFPP & FIB & 390 & 650 & 0.13 & 0.87 & & -1.00 & & ISSI \\
\hline \multirow[t]{2}{*}{ PtOEP } & GP-197 & 366,543 & 650 & 0.32 & 0.70 & 50.0 & -1.70 & Burns(1995) & Porphyrin \\
\hline & silica gel & & & 0.12 & 0.88 & & - & & \\
\hline Pyrene & $\begin{array}{ll}\text { GE } & \text { RTV } \\
118 & \end{array}$ & $360-390$ & 470 & 0.12 & 0.88 & & $\sim 0$ & & \\
\hline Ru(bpy) & silica gel & 337,457 & 600 & 0.33 & 0.68 & 3.0 & & Burns(1995) & Aldrich \\
\hline \multirow[t]{2}{*}{$\mathrm{Ru}\left(\mathrm{ph}_{2}-\mathrm{phen}\right)$} & silica gel & 337,457 & 600 & 0.17 & 0.84 & 4.7 & -1.30 & Burns(1995) & $\begin{array}{l}\text { GFS } \\
\text { Chem. }\end{array}$ \\
\hline & $\begin{array}{ll}\text { GE } & \text { RTV } \\
118 & \end{array}$ & & & 0.27 & 0.75 & & -0.78 & & \\
\hline$\left[\mathrm{Ru}\left(\mathrm{ph}_{2}-\text { phen }\right)_{3}\right]^{2+}$ & $\begin{array}{l}\text { GP- } \\
\text { 134/silica }\end{array}$ & 337 & 620 & 0.22 & 0.78 & 0.30 & & $\begin{array}{l}\text { Xu et al. } \\
(1994)\end{array}$ & \\
\hline \multirow[t]{2}{*}{ NASA-Ames PSP } & & & & 0.38 & 0.62 & & -1.50 & McLachlan & NASA \\
\hline & & & & & & & & $\begin{array}{l}\text { and Bell } \\
(1995)\end{array}$ & Ames \\
\hline McDonnell Douglas PSP & & blue & & 0.18 & 0.82 & & & Dowgwillo & McDonnell \\
\hline & & & & & & & & $\begin{array}{l}\text { et } \\
(1994)\end{array}$ & ח \\
\hline TsAGI LPSL2 & & $320-350$ & $425-550$ & 0.25 & 0.75 & & -0.30 & $\begin{array}{l}\text { Bukov et } \\
\text { al. (1993) }\end{array}$ & TsAGI \\
\hline
\end{tabular}





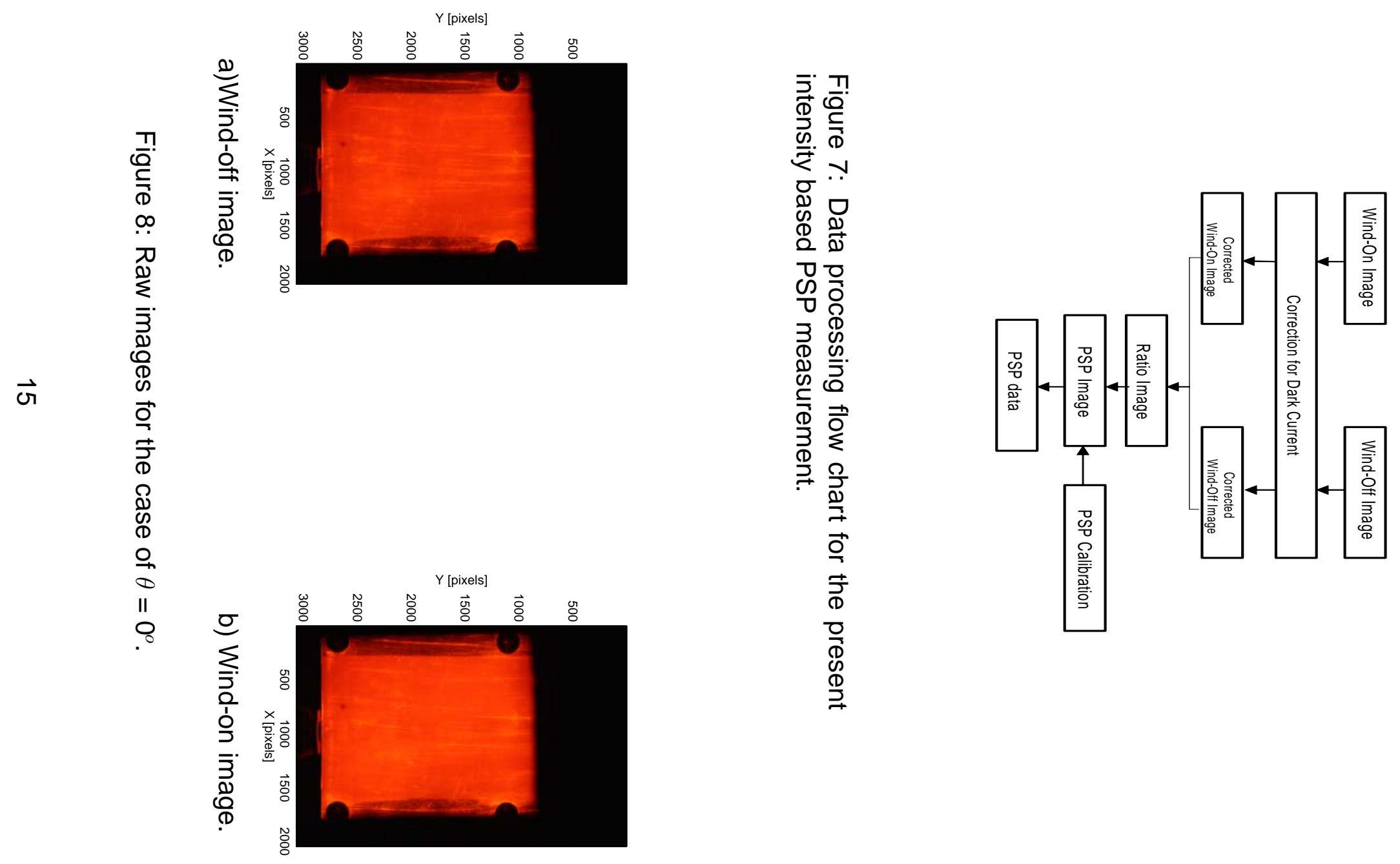


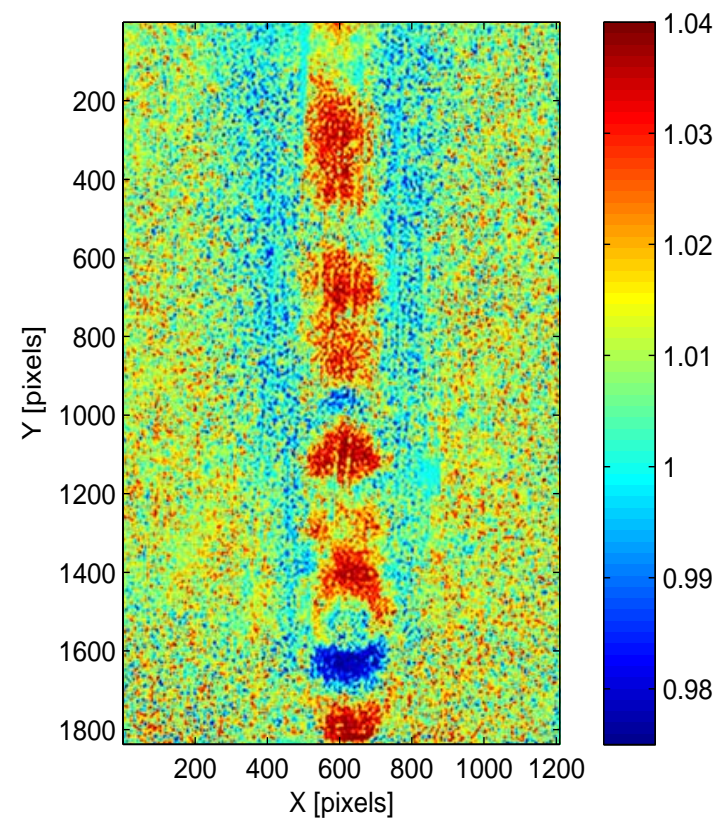

Figure 9: $I_{r e f} / I$ Post-processed image, $\theta=0^{\circ}$, nozzle center at $x=604$ pixels and $y=1800$ pixels. The nozzle diameter is equivalent $=185$ pixels

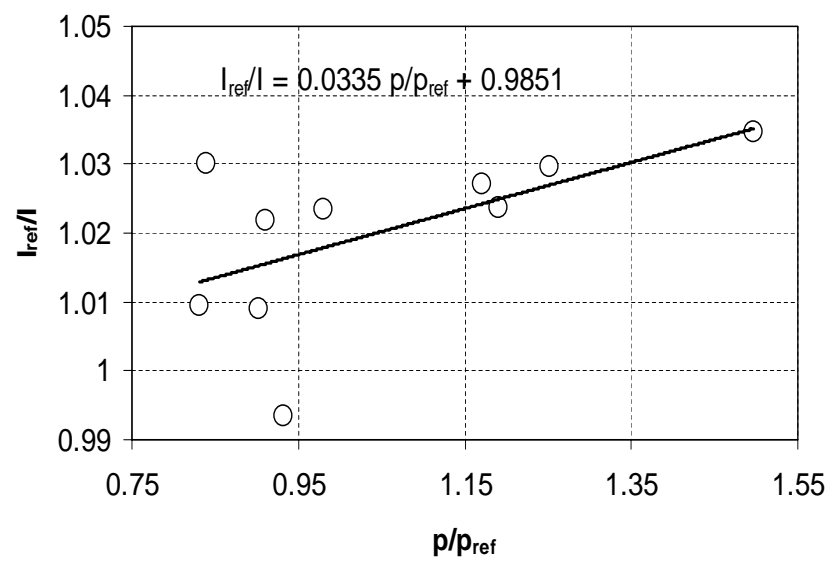

Figure 10: Calibration results by using in-situ technique, $\theta=0^{\circ}$. 


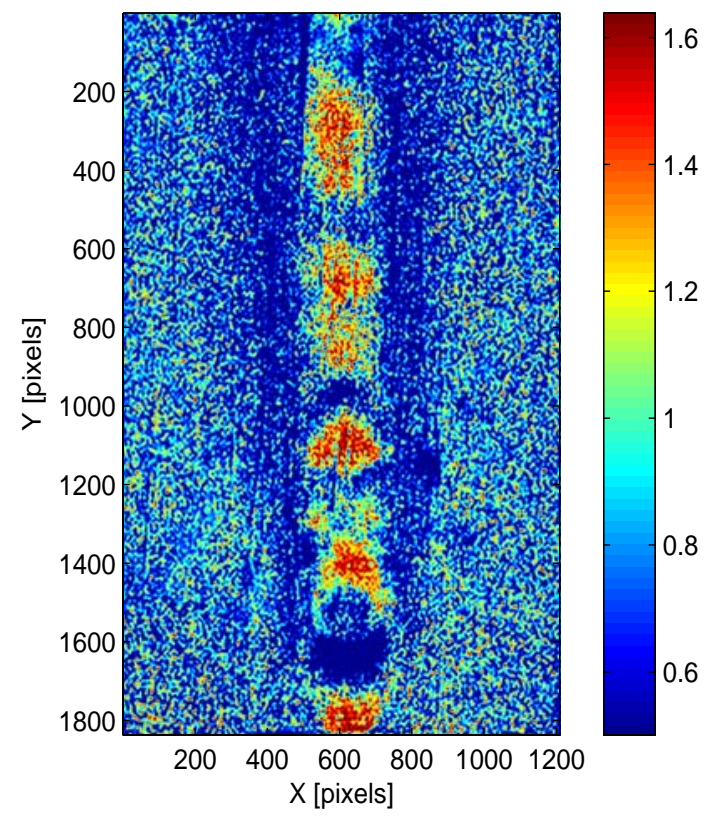

Figure 11: Calibrated image, $p / p_{\infty}, \theta=0^{\circ}$, nozzle center at $x=604$ pixels and $y=1800$ pixels. The nozzle diameter is equivalent $=185$ pixels

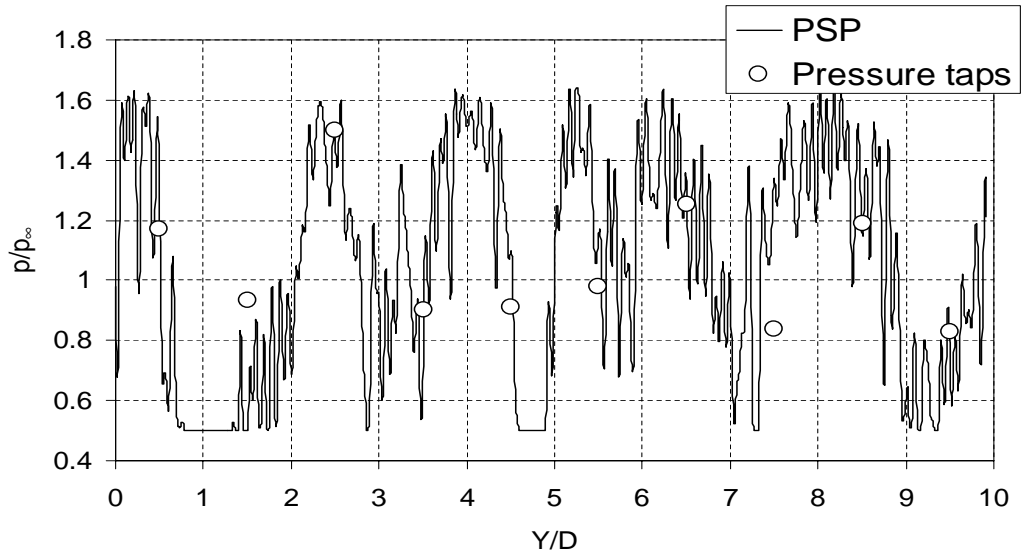

Figure 12: Plate centerline surface pressure distribution, $\theta=0^{\circ}$. 




Figure 13: $I_{\text {ref }} / I$ Post-processed image, $\theta=10^{\circ}$, nozzle center at $x=604$ pixels and $y=1800$ pixels. The nozzle diameter is equivalent $=185$ pixels



Figure 14: $I_{r e f} / I$ Post-processed image, $\theta=20^{\circ}$, nozzle center at $x=604$ pixels and $y=1800$ pixels. The nozzle diameter is equivalent $=185$ pixels 